based pharmaceutical company Novartis, which was approved for the treatment of chronic myelogenous leukaemia in 2001. Gleevec represents a new class of drugs that disrupt components of the intracellular signalling pathways that cause cells to grow and divide uncontrollably, giving rise to cancers.

There are more than 500 protein kinase genes in the human genome. They catalyse the specific phosphorylation of proteins and play an essential role in many signalling pathways, including those involved in cellcycle control.

One way of evaluating the substrate selectivity and function of protein kinases in a high-throughput format is by peptide microarrays to which the kinases will bind selectively. Pepscan Systems of Lelystad, the Netherlands, launched its PepChip Kinase peptide microarray about a year ago. "Currently, we have 1,150 different substrates," says Jos Joore, vice-president of array technology at Pepscan, all derived from a public database. The microarray can be used for substrate profiling of known and unknown kinases and for specificity testing of kinase inhibitors. The company is also turning its attention to proteases. Longer term, Joore says, it is trying to improve the specificity of its peptide substrates by providing them as constrained loops, or a combination of constrained loops, rather than linear peptides.

The three-dimensional surface of the PamChip from PamGene of 's-Hertogenbosch, the Netherlands, is designed to allow

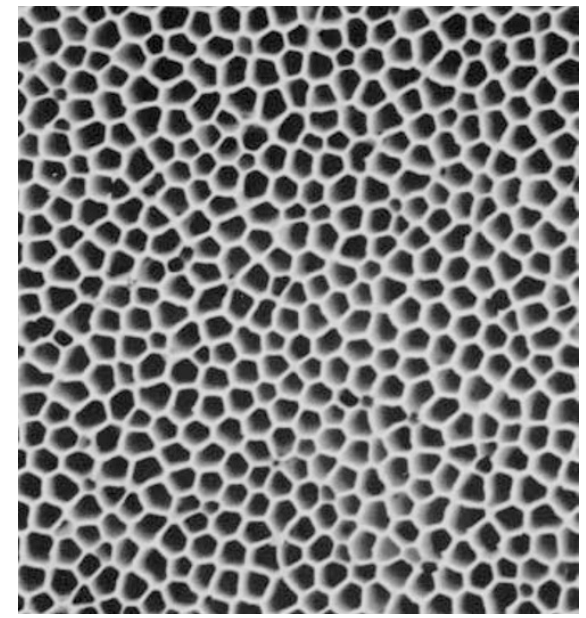

The porous PamChip surface provides a greater area for protein immmobilization.

peptide substrates to be deposited at higher concentrations than conventional arrays. "We can immobilize much more material per square millimetre than other flat materials," says Rob Ruijtenbeek, PamGene's head of kinase research. A 500-fold increase in reactive surface compared with two-dimensional arrays is claimed.

At the heart of the system is PamGene's 5D-Pulse flow-through microarray technology, in which peptides are covalently immobilized using inkjet technology onto the porous microarray surface via the peptide amino terminus. Sample is then pumped back and forth through the porous material to facilitate mixing. The pumping-cycle frequency can be changed and detection is with fluorescent antibodies using a CCD camera/microscope. The system provides kinetic readouts in which substrate conversion is monitored over time; traditional array formats limit detection to a single time point.

In June, PamGene announced plans to join forces with Jerini Peptide Technologies (JPT) in Berlin, Germany, which allows PamGene to marry its microarray platform with JPT's comprehensive peptide sets for kinase profiling. Zeptosens of Witterswil, Switzerland, is similarly increasing the range of its arrays by selling antibodies developed by Cell Signaling Technology of Beverly, Massachusetts, for use with the Zeptosens planar waveguide detection protein microarray platform. With this technology, only fluorophores located at or near the surface of the waveguide are excited and signals from unbound molecules in the bulk solution are not detected.

According to Peter Oroszlan, director of business development at Zeptosens, this technology provides a significant increase in signal-to-background ratios, enabling detection of low-abundance proteins such as signalling molecules. "Our system allows you to measure 600 protein molecules in a spot, which corresponds to one zeptomole [ $10^{-21}$ moles]," he says. ZeptoMARK protein microarrays are available in capture or reverse-array formats (see Nature 429, 102; 2004), and applications include expression monitoring of proteins during drug profiling and pathway mapping,

\title{
QUANTUM DOTS SHOW THEIR TRUE COLOURS
}

As an alternative to organic dyes, quantum dots (QDs) may be worth a closer look. These semiconductor nanometre-sized crystals, typically with a cadmium-based core, avoid some of the shortcomings associated with traditional organic dyes and fluorescent proteins (see Nature 413, 450; 2001). QDs are brighter, not prone to photobleaching, and come in a wide range of colours. By changing the size of the particle, the emission can be tuned to any

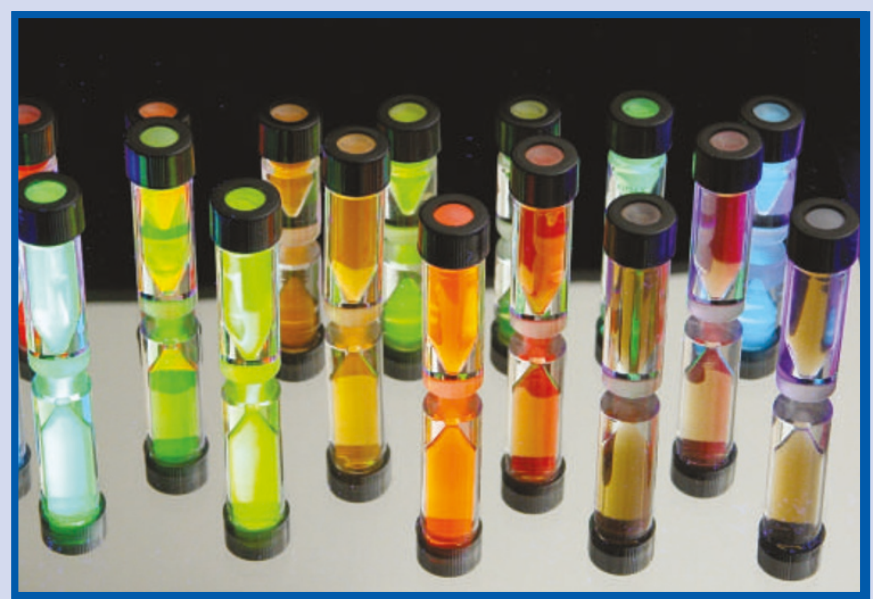

Evident Technologies' quantum dots come in many colours. wavelength, from ultraviolet to infrared. QDs also have narrow emission spectra, which means more colours can be used at a time with minimal channel overlap. Multiple colours of QDs can be simultaneously excited using a single light source. QDs have, for example, been used to to track the activity and diffusion of individual glycine receptors in the neuronal membrane for up to 20 minutes - considerably longer than is possible with Cy3 dyes.

"We don't expect QDs to completely displace organic dyes," says Clint Ballinger, chief executive of Evident Technologies in Troy, New York. "They make an attractive alternative for many different applications." Evident began by making ultrafast optical switches for the telecom industry. But when the bottom fell out of that business, the company turned to alternative applications for its nanocrystals. It sells its QDs (EviDots) as EviTags, which are water-stabilized, conjugation-ready QDs that can be coupled to proteins or antibodies, and as EviFluors, which have biotin groups.

Another company with know-how in this area is Quantum Dot Corporation (QDC) of Hayward, California. Its product line includes basic Qdot nanocrystals, Qdot bioconjugates coupled to proteins, oligonucleotides and small molecules (streptavidin, protein A and biotin), and Qbead microspheres. In June, QDC introduced a kit that enables researchers to conjugate their own antibodies to Qdot nanocrystals - a procedure that the company says typically takes 3-4 hours. The kit can also be used to couple other thiolcontaining molecules to QDs.

So what of the future? "Some customers don't like cadmium," says Ballinger, and Evident plans to launch indium phosphide-based EviDots in the next few months as an alternative to cadmium for the visible range. 